\title{
Exploring inside-out Doppler tomography: magnetic cataclysmic variables ${ }^{\star}$
}

\author{
E. J. Kotze ${ }^{1,2}$, S. B. Potter ${ }^{1}$, and V. A. McBride $\mathrm{e}^{1,2}$ \\ 1 South African Astronomical Observatory, PO Box 9, Observatory 7935, Cape Town, South Africa \\ e-mail: ejk@saao.ac.za \\ 2 Astrophysics, Cosmology and Gravity Centre (ACGC), Department of Astronomy, University of Cape Town, Private Bag X3, \\ 7701 Rondebosch, South Africa
}

Received 15 June 2016 / Accepted 25 August 2016

\begin{abstract}
Context. Doppler tomography of magnetic cataclysmic variables is a valuable tool for the interpretation of the complex spectroscopic emission line profiles observed for these systems.

Aims. We present the results of applying our inside-out velocity projection and flux modulation mapping techniques to the Doppler tomography of magnetic cataclysmic variables.

Methods. The inside-out projection reverses the standard velocity direction by transposing the zero-velocity origin to the outer circumference and the maximum velocities to the origin of the velocity space. The inside-out tomogram is constructed by directly projecting phase-resolved spectra onto the inside-out framework. In addition, our flux modulation mapping technique extracts any information related to the modulation of the line flux by utilising consecutive half-phase tomograms. We apply this to both the standard and the inside-out techniques.

Results. Our test cases, the polars HU Aqr and V834 Cen, and the intermediate polar PQ Gem, were chosen because of their known accretion characteristics, namely ballistic, magnetic and curtain dominated accretion, respectively. In all three cases the inside-out tomogram better exposes low-velocity emission details which are overly compacted in the standard tomogram. This is especially apparent for the mid-inclination V834 Cen where the almost blob-like blended lower velocity emission in the standard tomogram is more exposed in the inside-out tomogram, making it easier to distinguish between the ballistic and magnetically confined accretion flows that are evident in the trailed spectra. Similarly, the inside-out tomogram enhances high velocity emission details which are washed out in the standard tomogram. This is particularly effective in revealing the high velocity magnetic accretion flows in the polars HU Aqr and V834 Cen. The addition of our flux modulation technique gives a significant improvement in reproducing the trailed input spectra adding more confidence to the interpretation of the Doppler maps. Furthermore, amplitude and phase maps are constructed that further reveal amplitude and phasing characteristics of the emission components in the three test cases.
\end{abstract}

Key words. accretion, accretion disks - techniques: spectroscopic - novae, cataclysmic variables - binaries close stars: magnetic field

\section{Introduction}

Cataclysmic variables (CVs) are interacting, semi-detached binary systems which consist of a white dwarf (primary) and a lower main-sequence red dwarf (secondary). The secondary fills its Roche lobe and loses material through the inner Lagrangian point $\left(L_{1}\right)$ of the system. This material flows in a free fall trajectory towards the primary and it may form an accretion disc around the primary before it is finally accreted. In magnetic CVs (mCVs) the magnetic field of the primary is sufficiently strong to disrupt completely or partially the formation of an accretion disc. Instead, part of the accretion flow is threaded along magnetic field lines to the poles of the primary in an accretion stream or an accretion curtain. A distinction is made between two classes of mCVs, namely polars and intermediate polars (IPs). In polars the primary's magnetic field is sufficiently strong to lock the primary into synchronous rotation with the binary orbit and to prevent completely an accretion disc from forming. In IPs the magnetic field of the primary is less strong and the primary

* A copy of the code is available at the CDS via anonymous ftp to cdsarc.u-strasbg. fr (130.79.128.5) or via

http://cdsarc.u-strasbg.fr/viz-bin/qcat?]/A+A/595/A47 rotates asynchronously with the binary orbit. The formation of a disc may be completely or partially disrupted. Hellier (2001) and Warner (2003) provide comprehensive reviews of all classes of CVs and mCVs. See also Ferrario et al. (2015) for an extensive review on the current observational and theoretical research of isolated and binary magnetic white dwarfs.

Doppler tomography was introduced by Marsh \& Horne (1988). It was initially developed as a technique which uses orbitally phase-resolved spectra to construct a two-dimensional tomogram (velocity image) of the accretion disc of a CV. Doppler tomography has revolutionised the interpretation of complex line profiles observed in the phase-resolved spectra of interacting binary systems such as CVs and mCVs (see, e.g. Marsh 2001). One of the seminal results of Doppler tomography applied to mCVs was presented by Schwope et al. (1997). They were able to identify three different emission line components, with different width and radial velocity variation, in the observed spectra of the polar HU Aqr. They then used Doppler tomography to locate the origin of these components (1) on the secondary; (2) in the ballistic; and (3) in the magnetically confined parts of the accretion flow. Amongst other examples, Šimić et al. (1998) and 
Potter et al. (2004) were able to do the same for V2301 Oph and V834 Cen, respectively. Hellier (1997, 1999) used spin-cycle Doppler tomography to map the spin-varying emission components in intermediate polars. It was especially successful in exposing the extent and shape of the line-emitting accretion curtains in, for example, PQ Gem (Hellier 1997, 1999).

Modulation Doppler tomography, a notable extension to the standard technique, was introduced by Steeghs (2003). The technique is able to isolate emission components which vary harmonically as a function of the orbital (or spin) period. This is achieved through the simultaneous construction of three tomograms, that is, one for the average flux distribution and two for the variable part in terms of its sine and cosine amplitudes. Recent references in literature to the application of this technique mostly involve spectroscopic studies of black hole X-ray binaries, for example, Peris et al. (2015) and Čechura et al. (2015).

Inside-out Doppler tomography, a complementary extension to the standard technique, was introduced by Kotze et al. (2015, hereafter Paper I). This technique reverses the velocity axis to create an inside-out Doppler coordinate frame. Inside-out tomograms are constructed independently of the corresponding standard tomograms. This is achieved by directly projecting phaseresolved spectra onto the inside-out framework. The objective was to explore a more intuitive velocity layout for Doppler tomograms that rectified their inside-out appearance. The results presented in Paper I also showed that the inside-out projection can enhance high velocity emission details which are washed out in the standard projection. Similarly, it can expose low-velocity emission details which are overly compacted.

This paper expands on the preliminary results presented in Potter et al. (2016). We apply our inside-out projection technique and a variant form of modulation Doppler tomography to the spectroscopic observations of mCVs. First, in Sect. 2 we review the polar coordinate frame for the standard and the insideout velocity space previously introduced. Next, in Sect. 3 we do a comparison between the standard and inside-out tomograms of three mCVs, namely the polars HU Aqr and V834 Cen, as well as the intermediate polar PQ Gem. We investigate how the relative contrast levels amongst emitting components such as the ballistic and magnetically confined accretion flows are redistributed by the inside-out framework. In Sect. 4 we present a variant form of modulation Doppler tomography. Our flux modulation mapping technique extracts any phased modulation in the observed flux from consecutive half-phase standard and inside-out tomograms. We provide a summary and conclusions in Sect. 5.

\section{Velocity space}

We first review a polar coordinate frame that co-rotates with a $\mathrm{mCV}$ with ballistic and magnetic accretion flows only. Paper I asserted that a polar coordinate frame is more conducive to the circularly symmetric nature of Doppler tomograms. It gives a more direct indication of velocities and directions, that is to say, describing velocity in terms of its magnitude and direction is more intuitive than describing it in terms of its $x$ - and $y$-axial components.

The top panel in Fig. 1 shows the spatial polar coordinates for a model $\mathrm{mCV}$ without an accretion disc, but with a ballistic stream and magnetically confined accretion along the magnetic dipole field lines. The spatial coordinates are the radial distance $r$ from the origin, that is, the binary's centre of mass (C.O.M.), and the polar angle $\theta_{s}$ (not to be confused with $\theta$ in the velocity frame) measured in an anti-clockwise direction from the line between the binary's C.O.M. and the secondary. The $r$ coordinate is normalised by the binary separation $a$. The orbital motion is counter-clockwise and the point of mid-eclipse of the primary by the secondary is defined as the binary orbital phase zero.

The middle panel in Fig. 1 shows the corresponding velocity map in standard polar coordinates. The velocity magnitude $v$ increases linearly outwards from the origin and the velocity direction is the polar angle $\theta$ measured anti-clockwise from the line drawn from the origin horizontally to the right (i.e. the $0^{\circ}$-line). For example, the secondary has a velocity magnitude of $\sim 400 \mathrm{~km} \mathrm{~s}^{-1}$ with a velocity direction (angle) of $90^{\circ}$. The increasing velocity of the ballistic part of the accretion stream is reflected in the outwards curve of the velocity profile from where it leaves the secondary towards higher velocities. Similarly, the profiles of the dipole trajectories radiate outwards from the lower velocities where they leave the orbital plane towards higher velocities as they fall down onto the primary, in other words, they diverge towards higher velocities.

The bottom panel in Fig. 1 shows the inside-out velocity map. In the inside-out velocity polar coordinate frame zero velocity is on the outer circumference and the maximum velocities are around the centre of the coordinate frame. In order to provide a complementary view to the standard framework the centre of the inside-out framework is set to the maximum radial velocity extracted from the phase-resolved spectra, that is to say, the 'edge' of the data projected onto the framework to construct the tomogram. However, we note that the centre of the framework represents a discontinuity and, therefore, the inner four velocity bins (pixels) around it are ignored. The velocity magnitude $v$ now increases linearly inwards from zero velocity towards the origin, while the velocity direction is still given by the polar angle $\theta$ measured anti-clockwise from the $0^{\circ}$-line. The profile of the secondary is now upside down since it is orbiting as a solid body with the outside moving faster than the inside. The outer bounded circular ring area, between zero velocity and the dashed line, is the Roche lobe of the primary. As one can see in the inside-out framework the profile of the ballistic stream now curves inwards as it accelerates towards higher velocities. The profiles of the dipole trajectories now radiate inwards from the lower velocities where they leave the orbital plane towards higher velocities as they fall down onto the primary, in other words, they converge towards higher velocities.

\section{Doppler tomography: standard and inside-out projections}

All the standard and inside-out Doppler tomograms presented hereafter were constructed using our fast maximum entropy (FME) Doppler tomography code. Our code is based on the FME code presented by Spruit (1998) and it can construct tomograms in the standard or the inside-out velocity coordinate frame. The inside-out tomogram is constructed independently of the standard tomogram by directly projecting phase-resolved spectra onto the inside-out velocity space.

All the tomograms are shown with model velocity overlays. These include the Roche lobes of the primary and the secondary as well as a single particle ballistic trajectory from the $L_{1}$ point representing the ballistic accretion flow. The single particle ballistic trajectory includes gravitational and centrifugal effects. Also included in the ballistic trajectory is a magnetic drag force which decreases exponentially as a function of radius. This drag force is similar to that described by Schwope et al. (1997, 2000), 


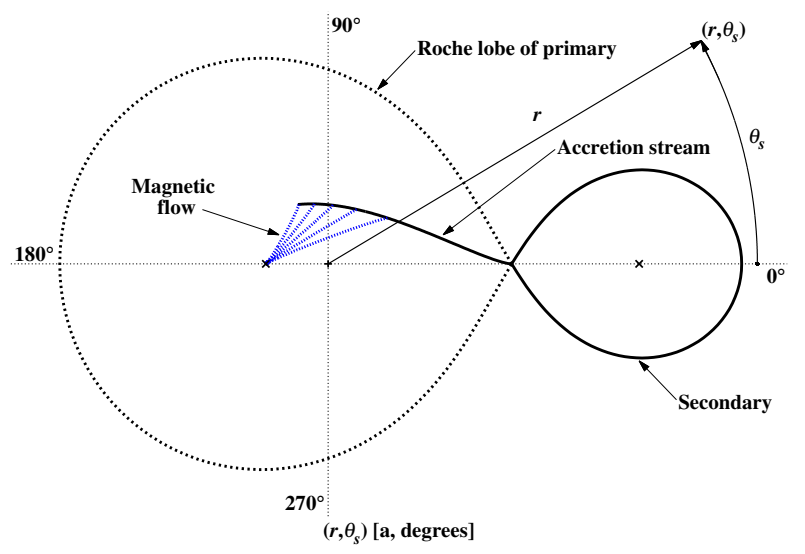

$90^{\circ}$


Fig. 1. Standard spatial and velocity, and inside-out velocity polar coordinates for a model mCV with ballistic and magnetic accretion flows only. The top and middle panels show the standard spatial and velocity polar coordinate layout, respectively. The bottom panel shows the inside-out velocity polar coordinate layout. The assumed binary system parameters for the model are: a primary mass of $0.8 M_{\odot}$; a mass ratio ( $q$ ) of 0.2 ; an orbital period of $0.083333 \mathrm{~d}(\sim 120 \mathrm{~min})$ and an inclination of $87^{\circ}$. The C.O.M. of the binary is marked with a plus (+) while that of the primary and secondary are marked with crosses $(\times)$. Overlays are shown for the Roche lobe of the primary, the accretion stream from the secondary towards the primary (up to an azimuth angle of $60^{\circ}$ ), as well as for magnetic dipole field lines at $10^{\circ}$ intervals from $20^{\circ}$ to $60^{\circ}$ in azimuth around the primary. The assumed dipole axis azimuth and co-latitude are $35^{\circ}$ and $15^{\circ}$, respectively.

and it is included in order to account for the pull of the primary's magnetic field on the partly ionised stream. The model velocity overlays also include some single particle magnetic dipole trajectories representing the magnetically confined accretion flow.
Our test cases were selected for the distinct accretion features that are apparent in their trailed spectra and exposed through standard Doppler tomography. In the standard tomogram of the eclipsing polar HU Aqr (Schwope et al. 1997) the emission associated with a ballistic accretion stream is very prominent. On the other hand, emission associated with a magnetically confined accretion flow is prominent in the standard tomogram of the non-eclipsing polar V834 Cen (Potter et al. 2004). The standard spin-cycle tomograms of the intermediate polar PQ Gem (Hellier 1997, 1999) exclusively show emission associated with a magnetically confined accretion flow locked on the spin period of the primary.

The validity and usefulness of Doppler tomograms depend on how well features in the observed spectra are reproduced in the reconstructed spectra. The results from our test cases show that normal Doppler tomography (standard and inside-out projections) is able to reproduce the basic structure of the observed spectra as well as most of the features linked to the prominent emission components. However, some features of the more complex structures are not fully reproduced in all cases. This, in part, is due to the assumption that Doppler tomography presents an average phase map and does not take into account the flux variations phased with the period. In Sect. 4 we show that our flux modulation mapping technique has the ability to improve the reconstruction of the observed spectra.

For all the test cases we show normalised standard and inside-out tomograms, as well as normalised trailed input, reconstructed and absolute input-minus-reconstructed $(\mathrm{O}-\mathrm{C})$ residual spectra. The input and reconstructed spectra are normalised by the maximum flux in the input spectra. We choose to show absolute $\mathrm{O}-\mathrm{C}$ spectra in order to have all the displayed trailed spectra on a comparable colour scale and calculate a simple root mean square (rms) value for the residuals, that is,

$\mathrm{rms}=\sqrt{\frac{1}{n} \sum_{i=1}^{n}\left(\mathrm{O}_{i}-\mathrm{C}_{i}\right)^{2}}$,

where $n$ is the total number of data points. All the tomograms, standard and inside-out, were constructed using 3996 discrete velocity bins (pixels). We choose a slightly higher value for the regularisation parameter $\alpha$, that is, 0.003 as opposed to the default value of $\sim 0.002$, as described by Spruit (1998). This was used for all the tomograms so that all are on the same comparable measure of regularisation. Also, it produces solutions that reproduce the detail in the observed spectra, but it does not overfit the noise.

\subsection{The polar HU Aqr}

HU Aqr is a bright eclipsing polar. Standard Doppler tomography of HU Aqr presented by Schwope et al. (1997) showed an extraordinary prominent ballistic stream, at least compared to other polars, distinct emission from the secondary and a diffuse patch of emission thought to be associated with the magnetically confined accretion flow. HU Aqr is therefore an excellent candidate as a test case for inside-out Doppler tomography. Figure 2 shows the results of applying standard and inside-out Doppler tomography to the He II (14686) emission line from $1993 \mathrm{Au}-$ gust spectroscopic observations of the high accretion state of $\mathrm{HU}$ Aqr. These observations were presented first by Schwope et al. (1997) and the reader is referred to that paper for a description of the data set and reduction procedures. Because HU Aqr is an eclipsing system, we exclude a small range around phase 0.0 


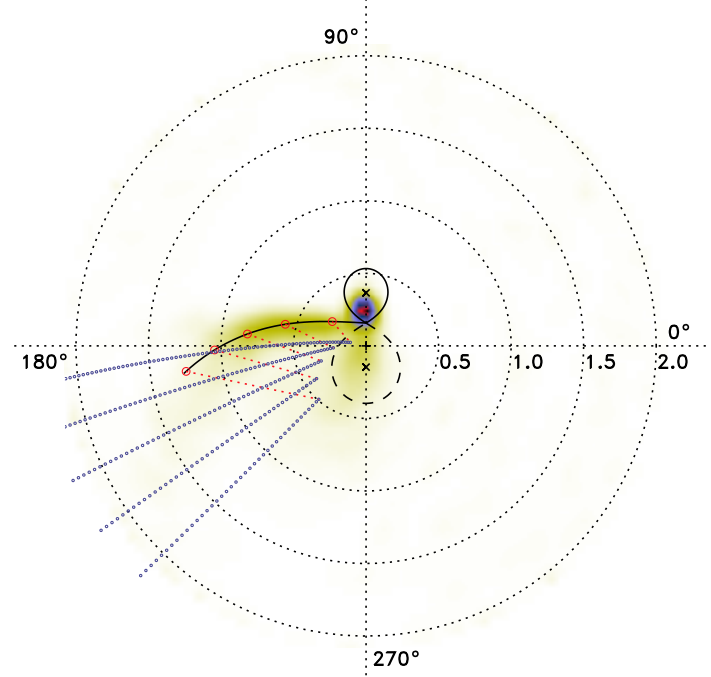

$(v, \theta)\left[10^{3} \mathrm{~km} \mathrm{~s}^{-1}\right.$, degrees $]$

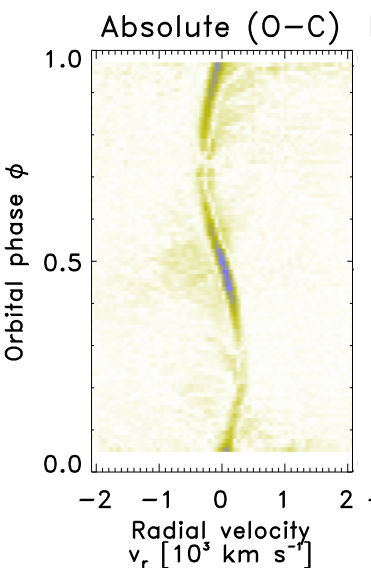



$(v, \theta)\left[10^{3} \mathrm{~km} \mathrm{~s}^{-1}\right.$, degrees $]$

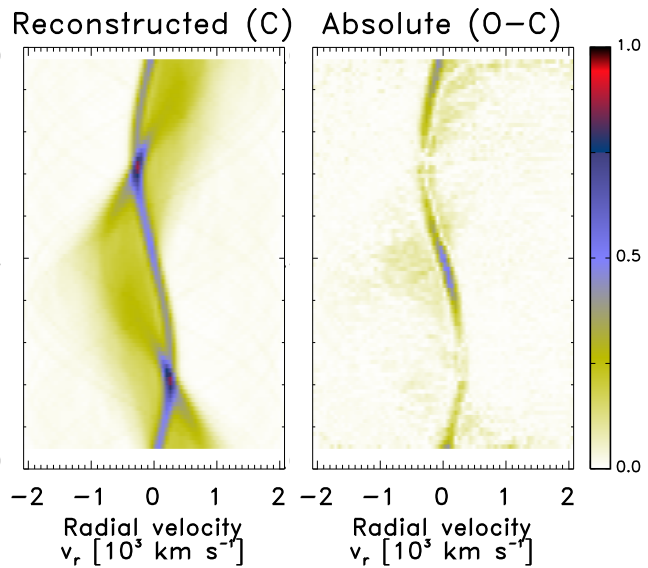

Fig. 2. Doppler tomography of HU Aqr. The standard and inside-out tomograms are shown top left and right, respectively. The bottom middle panel shows the trailed input spectra. The panels to the left and right of the middle panel show the trailed reconstructed and absolute residual spectra for the corresponding tomogram. The model velocity profile overlay shown in both tomograms is based on one of the models used by Schwope et al. (1997) with an inclination angle of $84^{\circ}$, a primary mass of $0.875 M_{\odot}$, a mass ratio $q=0.40$ and an orbital period of $0.086820446 \mathrm{~d}$ ( $\sim 125 \mathrm{~min}$ ). The overlay includes the Roche lobes of the primary (dashed line) and the secondary (solid line) as well as a single particle ballistic trajectory from the $L_{1}$ point up to $45^{\circ}$ in azimuth around the primary (solid line). Magnetic dipole trajectories are calculated at $10^{\circ}$ intervals from $5^{\circ}$ to $45^{\circ}$ in azimuth around the primary (thin dotted lines). The first dipole trajectory starts at $\left(123 \mathrm{~km} \mathrm{~s}^{-1}, 167^{\circ}\right)$, with consecutive trajectories starting at locations with progressively higher velocities and polar angles. The first dipole connection (small circles) is at $\left(340 \mathrm{~km} \mathrm{~s}^{-1}, 145^{\circ}\right)$. The dipolar axis azimuth and co-latitude, as modelled by Heerlein et al. (1999), are $\sim 38^{\circ}$ and $\sim 12^{\circ}$, respectively.

and only use the 92 spectra covering the orbital phase range 0.06-0.97. The spectra are evenly spaced and each spectrum has 72 velocity points in the extracted radial velocity range around the He II ( 24686$)$ emission line.

The trailed input spectra show three distinct emission components. The first component is the prominent narrow line which is brightest around phase 0.5 and which has zero radial velocity at phase 0.0. This component has a low-velocity amplitude and is associated with emission from the irradiated secondary. The second component is also relatively narrow, but it has a high velocity amplitude. It has a maximum redshift around phase 0.95 and a maximum blueshift around phase 0.45 . This component crosses the first component around phases 0.2 and 0.7 . The third component is relatively broad and visible throughout the covered phase range. It has a maximum blueshift around phase 0.40 . Both the second and third component are associated with emission produced in different parts of the accretion flow.

The emission from the irradiated secondary is seen as a bright spot at $\left(360 \mathrm{~km} \mathrm{~s}^{-1}, 90^{\circ}\right)$ in both the standard and inside-out tomograms. This feature in the tomograms is well traced by the velocity profile of the Roche lobe of the secondary. In the standard tomogram the secondary dominates the brightness scale due to its compacted projection. In contrast, its more extended projection in the inside-out tomogram allows other emission features to appear brighter.

In the standard tomogram the emission associated with the ballistic part of the accretion stream is a prominent, slightly downward-sloping horizontal ridge with an apparent relative constant brightness. However, a closer inspection reveals that at first, as the stream leaves $L_{1}$ at $\left(200 \mathrm{~km} \mathrm{~s}^{-1}, 90^{\circ}\right)$, it is faint, but it becomes brighter as it reaches $\left(300 \mathrm{~km} \mathrm{~s}^{-1}, 135^{\circ}\right)$. It keeps a relative consistent brightness up to $\left(750 \mathrm{~km} \mathrm{~s}^{-1}, 170^{\circ}\right)$ before it starts to become fainter. It stays clearly discernible up to $\left(1000 \mathrm{~km} \mathrm{~s}^{-1}\right.$, $\left.180^{\circ}\right)$. After this point it becomes more diffused and almost no detail is discernible at velocities above $1000 \mathrm{~km} \mathrm{~s}^{-1}$. This distribution in brightness along the ballistic stream is more prominent in the inside-out tomogram, that is, the lower velocity part is even fainter, whereas the mid- and high-velocity parts 
are significantly brighter. The lower velocity emission appears fainter as the emission is re-binned in a larger area with more pixels. Conversely, the high velocity emission is re-binned in a smaller area with fewer pixels. The mid-velocity emission, especially in the range $\left(500-1000 \mathrm{~km} \mathrm{~s}^{-1}, 160-180^{\circ}\right)$, appears brighter because overall the relative contrast levels changed due to the more extended projection of the secondary. In both tomograms the brighter part of the ballistic stream is well traced by the modelled velocity profile for the threading region, that is, the region where the ballistic stream is threaded onto the magnetic field lines, but before it leaves the orbital plane. Modelling done by Heerlein et al. (1999) on the magnetic stripping of the ballistic stream showed that it is possible for the stream to "survive" up to a distance of $0.5 a$ along its trajectory. This is equivalent to a velocity of $\sim 1250 \mathrm{~km} \mathrm{~s}^{-1}$. It is therefore possible that part of the emission visible in the inside-out tomogram along the extended ballistic trajectory in the velocity range $\left(1000-1500 \mathrm{~km} \mathrm{~s}^{-1}, 180-220^{\circ}\right)$ can be ascribed to the ballistic stream. This tenuous higher velocity $\left(>1000 \mathrm{~km} \mathrm{~s}^{-1}\right)$ emission is not clearly discernible in the standard tomogram. Resolving it in the inside-out tomogram is possible as the emission is re-binned in a smaller area with fewer pixels.

The diffuse patch of emission seen in the standard tomogram in the velocity range $\left(0-500 \mathrm{~km} \mathrm{~s}^{-1}, 180-270^{\circ}\right)$ is considered to be associated with the magnetically confined accretion flow that has left the orbital plane. In the inside-out tomogram this patch of emission is exposed to extend to even higher velocities, covering a velocity range of $\left(0-1000 \mathrm{~km} \mathrm{~s}^{-1}, 180-270^{\circ}\right)$.

The three distinct line components identified in the input spectra are reproduced fairly well in the reconstructed spectra from both the standard and inside-out tomograms. However, neither of the projections is able to reproduce the observed flux distribution in the prominent narrow line component associated with emission from the irradiated secondary. This is clearly seen in both sets of absolute $\mathrm{O}-\mathrm{C}$ spectra. Overall, the two projections performed equally well in reproducing the input spectra, that is, the residuals of both have rms values of $0.043(n=6624)$. In Sect. 4.1 we show the improvement in the reproduction of the narrow feature achieved with our flux modulation mapping technique.

\subsection{The polar V834 Cen}

V834 Cen is a bright non-eclipsing polar. Potter et al. (2004) presented standard Doppler tomography of V834 Cen that exposed dominant emission from the secondary and (or) from around $L_{1}$, less discernible emission from the ballistic stream and extended diffuse emission considered to be consistent with the magnetically confined accretion flow. Figure 3 shows standard and inside-out Doppler tomography based on the He II ( 14686$)$ emission line from the 2000 August spectroscopic observations of V834 Cen presented by Potter et al. (2004). The reader is referred to that paper for more information on the data set and reduction procedures. We use all 59 spectra from the original data set, which covers the whole orbital phase range, for our calculations. Each spectrum covers 0.05 of the phase with some overlap and has 73 velocity points in the extracted radial velocity range around the He II ( 24686$)$ emission line.

The trailed input spectra have a complex structure with at least three emission components. There is a blended low- to midvelocity component which is brightest between phases 0.0-0.2. It is fainter, but separated between phases $0.2-0.55$, and again blended and brighter between phases 0.55-1.0. This component is associated with blended emission from the irradiated secondary and the low- to mid-velocity part of the ballistic accretion stream. Also discernible is a fainter high-velocity broad base component underlying the whole phase range. The broad base component is associated with emission produced in different parts of the accretion flow. There is also a striking brighter blueshifted wing that follows closely the high-velocity edge of the underlying broad base component between phases 0.4-0.55. This high-velocity component is associated specifically with emission produced in the magnetically confined accretion flow as it falls towards the primary.

In the standard tomogram the emission from the irradiated secondary is blended with emission from the ballistic stream. This overly compacted blended emission creates an extremely bright ridge in the velocity range $\left(250-500 \mathrm{~km} \mathrm{~s}^{-1}\right.$, $\left.90-150^{\circ}\right)$ and dominates the brightness scale of the standard tomogram. In the inside-out tomogram this blended emission is more separated: the secondary is seen as a diffused patch at $\left(340 \mathrm{~km} \mathrm{~s}^{-1}, 90^{\circ}\right)$, while the stream forms a brighter ridge along the model stream trajectory in the velocity range $\left(300-400 \mathrm{~km} \mathrm{~s}^{-1}, 115-140^{\circ}\right)$. Separating this lower velocity emission in the inside-out tomogram is possible as the emission is re-binned in a larger area with more pixels.

The emission associated with the magnetically confined accretion as it leaves the orbital plane in the threading region is seen in both tomograms in the velocity range $\left(0-500 \mathrm{~km} \mathrm{~s}^{-1}\right.$, $\left.170-230^{\circ}\right)$. In the inside-out tomogram, however, the separation between the lower velocity emission from the ballistic stream and that from the magnetically confined accretion is more pronounced. Also, in both tomograms the emission considered to be consistent with the magnetically confined accretion as it flows down to the primary is centred on the ridge of brightness covering the velocity range $\left(700-1000 \mathrm{~km} \mathrm{~s}^{-1}, 185-235^{\circ}\right)$. However, in the inside-out tomogram the funnelling of the emission as it falls towards the primary along the dipole trajectories, especially those with azimuth angles $35^{\circ}$ and $45^{\circ}$, is significantly more apparent than in the standard tomogram.

The basic structure of the emission components seen in the input spectra is reproduced in the reconstructed spectra from both the standard and inside-out tomograms. However, the more complex structure between phases $0.2-0.55$ and the observed flux distribution in the narrower component between phases $0.0-0.2$ and $0.55-1.0$ are not reproduced by either of the projections. The absolute $\mathrm{O}-\mathrm{C}$ spectra of both projections clearly show the incomplete reconstructed parts. Both projections, however, achieved the same level of overall success, that is, the residuals of both have rms values of $0.076(n=4307)$. We show in Sect. 4.2 that our flux modulation mapping technique is able to reproduce the complex structure and the flux distribution.

\subsection{The intermediate polar PQ Gem}

PQ Gem is a bright, low-inclination ( $i \sim 30^{\circ}$; Hellier 1997) intermediate polar. Hellier $(1997,1999)$ presented spin-cycle Doppler tomography of PQ Gem (see the mentioned papers for a complete description of the technique). Spin-cycle tomography is uniquely geared to produce velocity maps of the emission components that are locked on the spin period of the primary, for example the accretion curtains of the magnetically confined accretion flow falling onto the primary. Figure 4 shows standard and inside-out spin-cycle Doppler tomography based on the spin-folded He II ( (4686) emission line from the 1993 December spectroscopic observations of PQ Gem presented first by Hellier (1997). The reader is referred to that paper for a detailed 


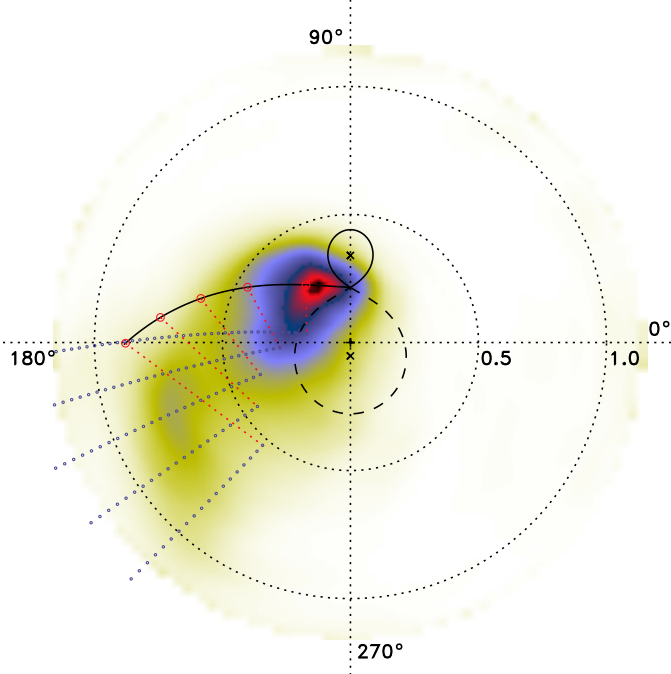

$(v, \theta)\left[10^{3} \mathrm{~km} \mathrm{~s}^{-1}\right.$, degrees $]$

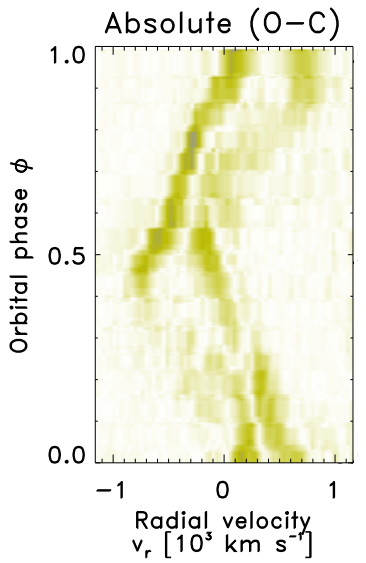

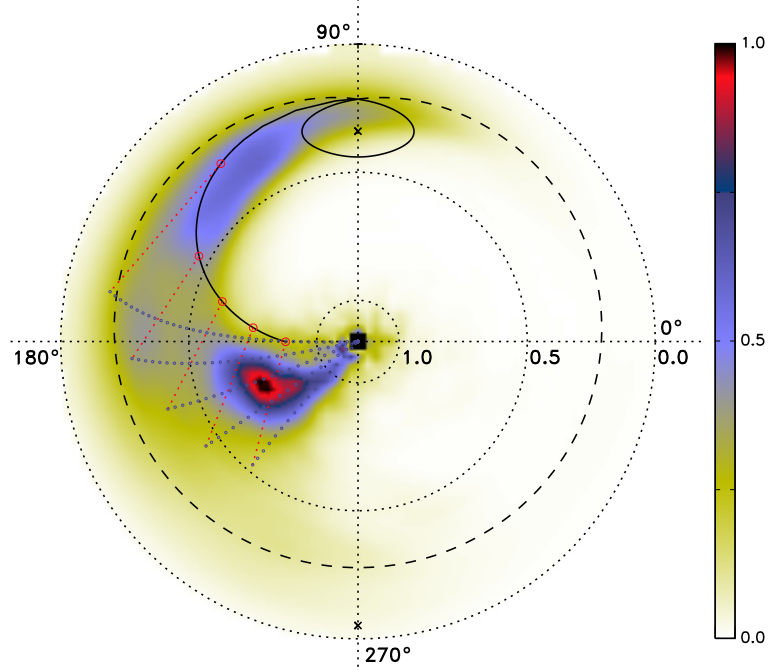

$(v, \theta)\left[10^{3} \mathrm{~km} \mathrm{~s}^{-1}\right.$, degrees $]$

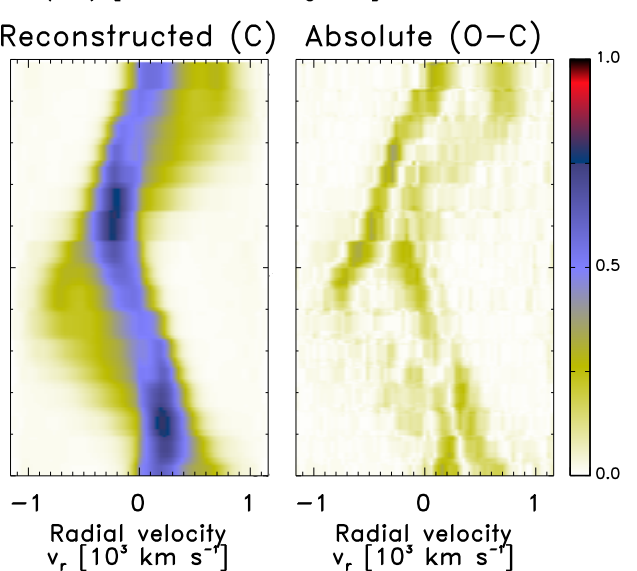

Fig. 3. Doppler tomography of V834 Cen. Same layout as Fig. 2. The model velocity profile overlay shown in both tomograms is based on the model used by Potter et al. (2004) with an inclination angle of $50^{\circ}$, a primary mass of $0.85 M_{\odot}$, a mass ratio $q=0.154$ and an orbital period of $0.070497518 \mathrm{~d}(\sim 101.5 \mathrm{~min}$; Schwope et al. 1993). The Roche lobes of the primary (dashed line) and the secondary (solid line) as well as a single particle ballistic trajectory from the $L_{1}$ point up to $45^{\circ}$ in azimuth around the primary (solid line), are included in the overlay. Magnetic dipole trajectories are calculated from $5^{\circ}$ to $45^{\circ}$ in azimuth around the primary (thin dotted lines) at $10^{\circ}$ intervals using a dipolar axis azimuth and co-latitude of $\sim 36^{\circ}$ and $\sim 20^{\circ}$ (Potter et al. 2004), respectively. The first of the dipole connections (small circles) are at $\left(360 \mathrm{~km} \mathrm{~s}^{-1}, 135^{\circ}\right.$ ) and the first dipole trajectory starts at $\left(150 \mathrm{~km} \mathrm{~s}^{-1}, 162^{\circ}\right)$. Consecutive trajectories start at locations with progressively higher velocities and polar angles.

description of the data set and reduction procedures. All 15 spectra from the original spin-folded data set, which covers the whole spin phase range, are used for our calculations. The spectra are evenly spaced and each spectrum has 361 velocity points in the extracted radial velocity range around the He II (24686) emission line.

At least two distinct emission components are seen in the spin-folded trailed input spectra. The first component has a maximum blueshift around phase 0.4 and a maximum redshift around phase 0.9 . It is bright at phases 0.3 and 0.65 , but the expected blue-to-red crossover is not clear. The second component has a lower velocity amplitude with a maximum redshift around phase 0.4 and a maximum blueshift around phase 0.9 . Although the red-to-blue crossover is visible for this component, the blueto-red crossover is again not clear. Both the components are associated with emission produced in the spin-locked magnetically confined accretion flow.

The standard tomogram of PQ Gem is dominated by two bright spots, one in the upper right quadrant and the other in the lower left quadrant. Hellier (1997, 1999) suggested that these two spots are associated with emission from the accretion curtains flowing towards, respectively, the lower and upper magnetic poles of the primary. The accretion curtains are considered to be formed by material at the magnetosphere flowing onto the magnetic field lines at an azimuth $30-50^{\circ}$ ahead of the accretion region on the primary (Hellier 1997). In the inside-out tomogram the spot seen in the upper right quadrant of the standard tomogram, is projected as an extended, more diffused feature. The brighter of the two spots seen in the standard tomogram, in other words, the one in the lower left quadrant, is also more extended, but still the most prominent feature.

The inside-out projection significantly changes the apparent flux distribution between these two emission features. This places greater emphasis on the difference in the velocities of the two features. If we consider the model velocity profile we find that in the inside-out projection the magnetic dipole lines converge towards higher velocities. This creates an intuitive representation of the curving accretion curtains as they fall down onto the primary. We note that the phase resolution (i.e. only 15 phase bins) of the data for PQ Gem may be considered inadequate to allow for any significant new insights. 


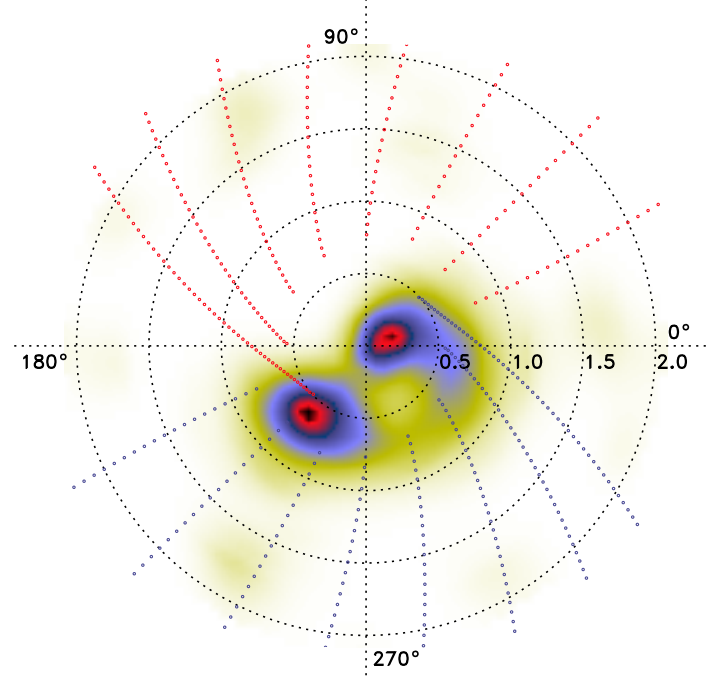

$(v, \theta)\left[10^{3} \mathrm{~km} \mathrm{~s}^{-1}\right.$, degrees $]$

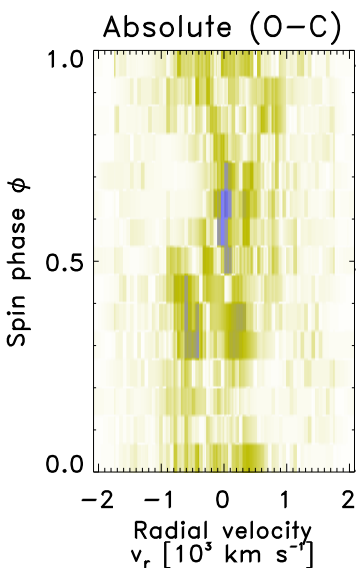

Reconstructed (C)

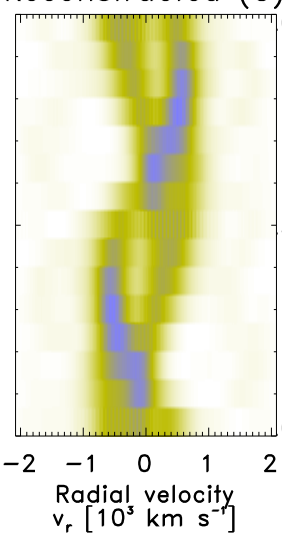

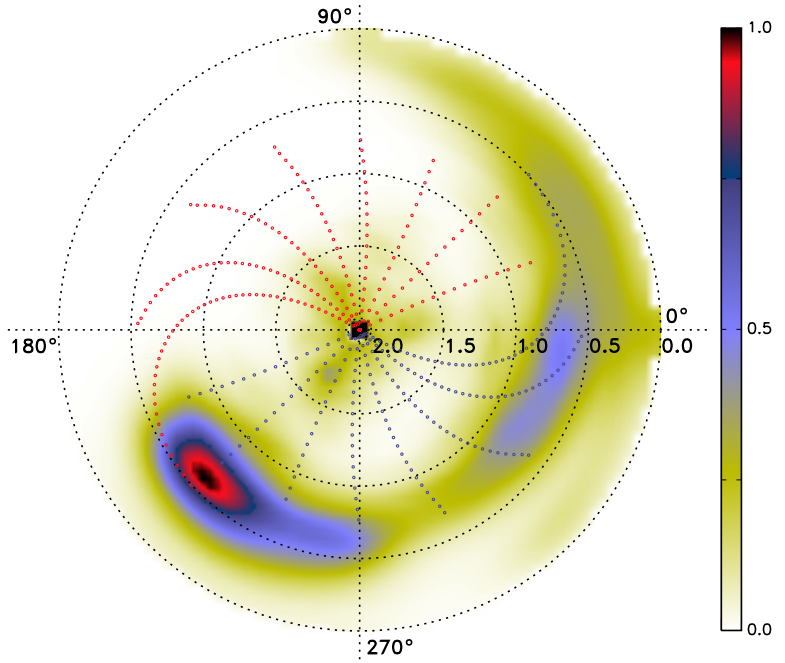

$(v, \theta)\left[10^{3} \mathrm{~km} \mathrm{~s}^{-1}\right.$, degrees $]$

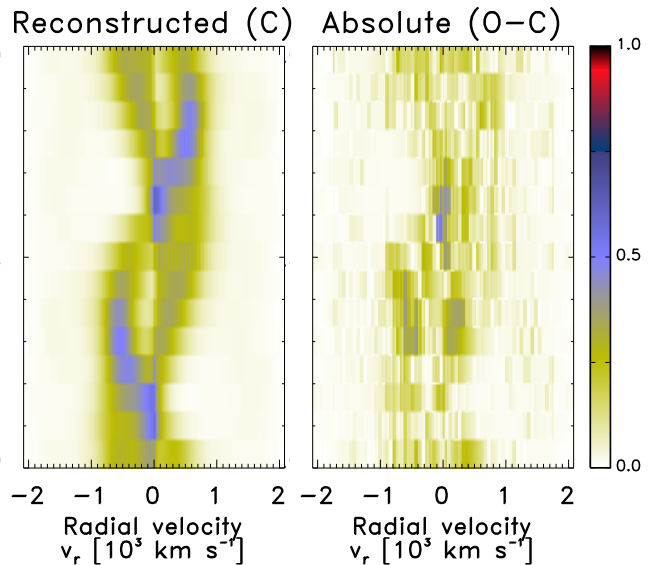

Fig. 4. Spin-cycle Doppler tomography of PQ Gem. The standard and inside-out spin-cycle tomograms are shown top left and right, respectively. The bottom middle panel shows the trailed input spectra. The panels to the left and right of the middle panel show the trailed reconstructed and absolute residual spectra for the corresponding spin-cycle tomogram. The model velocity profile overlay shown in both tomograms is based on an inclination angle of $30^{\circ}$ (Hellier 1997) and a dipolar axis co-latitude of $30^{\circ}$ (Potter et al. 1997). As proposed by Hellier (1997) we use a spin-wave velocity amplitude of $\sim 500 \mathrm{~km} \mathrm{~s}^{-1}$ and let the upper magnetic pole point towards us at spin phase 0.87 (dipolar axis azimuth $\sim 47^{\circ}$ ). We allow the upper curtain (thin blue dotted lines) to extend over spin phase $0.63-0.96$ and the lower curtain (thin red dotted lines) $0.13-0.46$.

The basic structure of the components identified in the input spectra is reproduced in the reconstructed spectra from both the standard and inside-out tomograms. However, the observed flux distribution, especially between phases $0.5-1.0$, is not reproduced by either of the projections. This partial reproduction is evident in the excess seen in both sets of absolute $\mathrm{O}-\mathrm{C}$ spectra. The inside-out projection achieved a marginal better overall result, that is, its residuals have a rms value of 0.092 compared to the 0.094 of the standard projection $(n=5415)$. In Sect. 4.1 we show how our flux modulation mapping technique improves the reproduction of the flux distribution.

\section{Doppler tomography: flux modulation mapping}

One of the axioms of Doppler tomography (Marsh 2001) is that the technique assumes that all points in the binary system being mapped are equally visible at all times. This implies that it is possible to construct a tomogram using spectra covering half of a phase only. The fact that this axiom is violated in most CVs and $\mathrm{mCVs}$ (especially in the higher inclination systems) actually enables us to selectively eliminate various emission components from the tomogram by using spectra from half of a phase only, thus revealing less obvious components (e.g. Potter et al. 2004).

Another axiom of Doppler tomography (Marsh 2001) is that the observed flux from any point in the binary system is constant. Observations of CVs and $\mathrm{mCVs}$, however, confirm that the flux from the typical emission components being mapped does modulate. Phased flux modulations are mostly attributed to the geometry of the system, for example, an eclipse or the aspect variation of an emission component. Doppler tomography, however, presents a phase-averaged map of the emission distribution in the system. Phase-dependent details in the observed spectra such as the orbital flux modulation will therefore not be recovered in the reconstructed spectra. Recognising this, Steeghs (2003) introduced modulation Doppler tomography, a technique that maps emission components which modulate sinusoidally as a function of the orbital (or spin) period. This is achieved through the simultaneous construction of three tomograms, that is, one for the average flux distribution and two for the variable part in terms of its sine and cosine amplitudes.

Exploiting the principles introduced by Steeghs (2003) and Potter et al. (2004) we present a variant form of modulation 
Doppler tomography. Our flux modulation mapping technique produces Doppler maps that represent the amplitude, phase and average of the modulated emission. By extracting any phased modulation in the observed flux from a series of consecutive half-phase tomograms it is possible to map how the flux from a specific emission component modulates over a complete phase. If it is assumed that the flux from an emission component varies harmonically over the observed phases then it is possible to extract the amplitude $\left(A_{j}\right)$, phase-offset $\left(\varphi_{j}\right)$ and average $\left(B_{j}\right)$ of the flux modulation in the $j$ th pixel from a simple sinusoidal fit of the form

$F_{i j}=A_{j} \sin \left[2 \pi\left(\Phi_{i}-\varphi_{j}\right)\right]+B_{j}$.

$F_{i j}$ and $\Phi_{i}$ are, respectively, the flux in the $j$ th pixel and the mid-phase value of the $i$ th half-phase tomogram. The fitted amplitude for each pixel $\left(A_{j}\right)$ produces an amplitude map which helps to identify which emission components are modulated. The normalised amplitude map is presented with the same colour scheme as the normal tomogram. Adding 0.25 to the fitted phaseoffset for each pixel $\left(\varphi_{j}\right)$ produces a map for the phase of maximum flux which shows at which phase an emission component appears brightest to an observer. This map is colour-coded according to phase: 0.0 - black, 0.25 - red, 0.5 - green and $0.75-$ blue. The average for each pixel $\left(B_{j}\right)$ is effectively the normal tomogram in the standard and inside-out projections. The flux modulation mappings presented hereafter are based on 10 consecutive half-phases (i.e. $0.0-0.5, \ldots, 0.4-0.9,0.5-0.0,0.6-0.1$, ..., 0.9-0.4). We also show normalised trailed summed input and reconstructed, as well as absolute $\mathrm{O}-\mathrm{C}$ spectra for all the test cases in a similar way as in the previous section.

\subsection{The polar HU Aqr}

Figure 5 shows standard and inside-out flux modulation mapping of HU Aqr. When compared with the reconstructed spectra in Fig. 2 the summed reconstructed spectra better reproduce the input spectra. Most striking is the almost complete absence of the bright narrow line flux between phases $0.0-0.2$ and $0.8-1.0$. This narrow line is associated with emission from the irradiated side of the secondary. It is expected to be absent in the range around phase 0.0 when the non-irradiated side of the secondary is pointing towards us as it eclipses the primary. The brightening around phase 0.5 in the same bright narrow emission is also slightly better reproduced. The improvement achieved for both projections is clearly seen in their absolute $\mathrm{O}-\mathrm{C}$ spectra with rms values of 0.026 compared to the non-modulated rms values of 0.043 .

In both the standard and inside-out amplitude maps we see that the secondary is the most flux modulated component. The flux from the ballistic stream is also shown to modulate, but more so in the inside-out map. HU Aqr is a high-inclination eclipsing system and we can therefore expect that the flux from the irradiated side of the secondary and the ballistic stream will modulate over the orbital phase of the system as these components get eclipsed.

In the phase of maximum flux maps the secondary is mostly green which indicates that it appears brightest around orbital phase 0.5 , when the irradiated side of the secondary is pointing towards us. The trailing and leading sides of the secondary are red and blue, respectively. This is expected since we have full views of the trailing and leading sides at orbital phases 0.5 and 0.75 , respectively. The ballistic stream is a mixture of blue and black which means it appears brightest between orbital phases
0.75 and 0.0 , in other words, between when we have a full view of the leading side of the stream and when the stream starts flowing away from us. The standard amplitude map and the phase of maximum flux map hold amplitude and phase information for the ballistic stream up to velocities of $\sim 500 \mathrm{~km} \mathrm{~s}^{-1}$ whereas the inside-out versions expose information up to velocities of $\sim 1000 \mathrm{~km} \mathrm{~s}^{-1}$.

\subsection{The polar V834 Cen}

The standard and inside-out flux modulation mapping results for V834 Cen are shown in Fig. 6. The summed reconstructed spectra better reproduce the input spectra when compared with the reconstructed spectra in Fig. 3. Most prominent is the considerable brightening in the narrower line flux between phases 0.0-0.2 and 0.55-1.0. Also, the more complex, multiple flux components between phases $0.2-0.55$, and the flux level of the striking high-velocity blue wing at phase $0.4-0.55$ are better reproduced. Both sets of absolute $\mathrm{O}-\mathrm{C}$ spectra clearly show the significant improvement achieved with rms values of 0.030 and 0.035 compared to the non-modulated rms values of 0.076 .

The most prominent flux modulated components in both the standard and inside-out amplitude maps are the ballistic and the magnetically confined accretion flows. In the inside-out amplitude map it almost looks like the ballistic stream is "split" into two parts, that is, one with velocities less than and the other with velocities greater than $250 \mathrm{~km} \mathrm{~s}^{-1}$. However, this is a neat separation of the magnetically confined accretion flow as it leaves the orbital plane along the early part of the ballistic stream (velocities $<250 \mathrm{~km} \mathrm{~s}^{-1}$ ) and the mid-velocity part of the "real" ballistic stream (velocities $>250 \mathrm{~km} \mathrm{~s}^{-1}$ ). The secondary is also flux modulated, but since V834 Cen is a mid-inclination noneclipsing system the amplitude of the modulation is not as dominant as that of the secondary of HU Aqr, which is an eclipsing system.

The real ballistic stream starts out as black in both the phase of maximum flux maps, then it switches to blue and a mixture of blue and green. This means that the lower velocity part of the stream appears brightest at orbital phase 0.0 when it is pointing away from us, while the mid-velocity part appears brightest at orbital phase 0.75 when we have a full view of the outer side of the stream. The phase of maximum flux interpretation for the higher velocity part of the ballistic stream is ambiguous since emission from this part is blended with emission from the magnetically confined accretion flow. The mid- to high-velocity part of the threading region is predominantly red in both maps, that is to say, it appears brightest at orbital phase 0.25 when we have a full view of the inner sides of the ballistic and magnetically confined accretion flows. The low-velocity part of the threading region, on the other hand, is black and red, which means that it appears brightest between orbital phases 0.0 and 0.25 when it is predominantly flowing away from us. The higher velocity part of the magnetically confined accretion flow is mostly green, in other words, it appears brightest around orbital phase 0.5 when we have a full view of the outer side of the magnetically confined accretion flow with most of it flowing towards us.

\subsection{The intermediate polar $P Q$ Gem}

Figure 7 shows standard and inside-out flux modulation mapping of PQ Gem. Compared to the reconstructed spectra in Fig. 4 the summed reconstructed spectra better reproduce the input spectra. Even though the flux levels between phases $0.25-0.75$ are not 


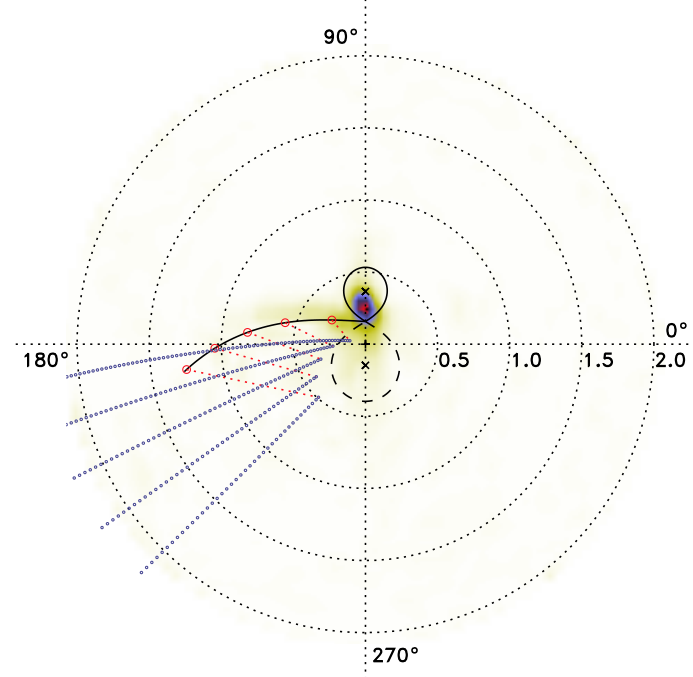

$(\mathrm{v}, \theta)\left[10^{3} \mathrm{~km} \mathrm{~s}^{-1}\right.$, degrees $]$

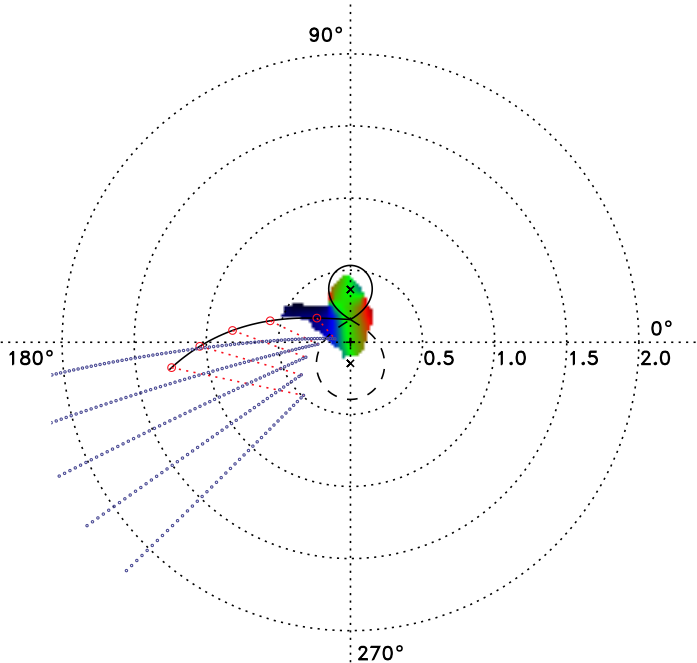

$(\mathrm{v}, \theta)\left[10^{3} \mathrm{~km} \mathrm{~s}^{-1}\right.$, degrees $]$



$(\mathrm{v}, \theta)\left[10^{3} \mathrm{~km} \mathrm{~s}^{-1}\right.$, degrees $]$

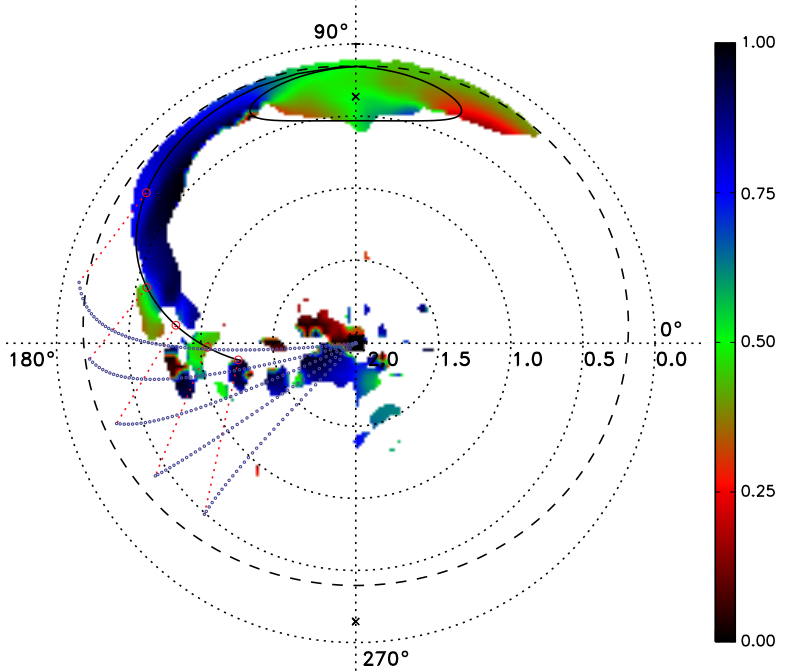

$(v, \theta)\left[10^{3} \mathrm{~km} \mathrm{~s}^{-1}\right.$, degrees $]$
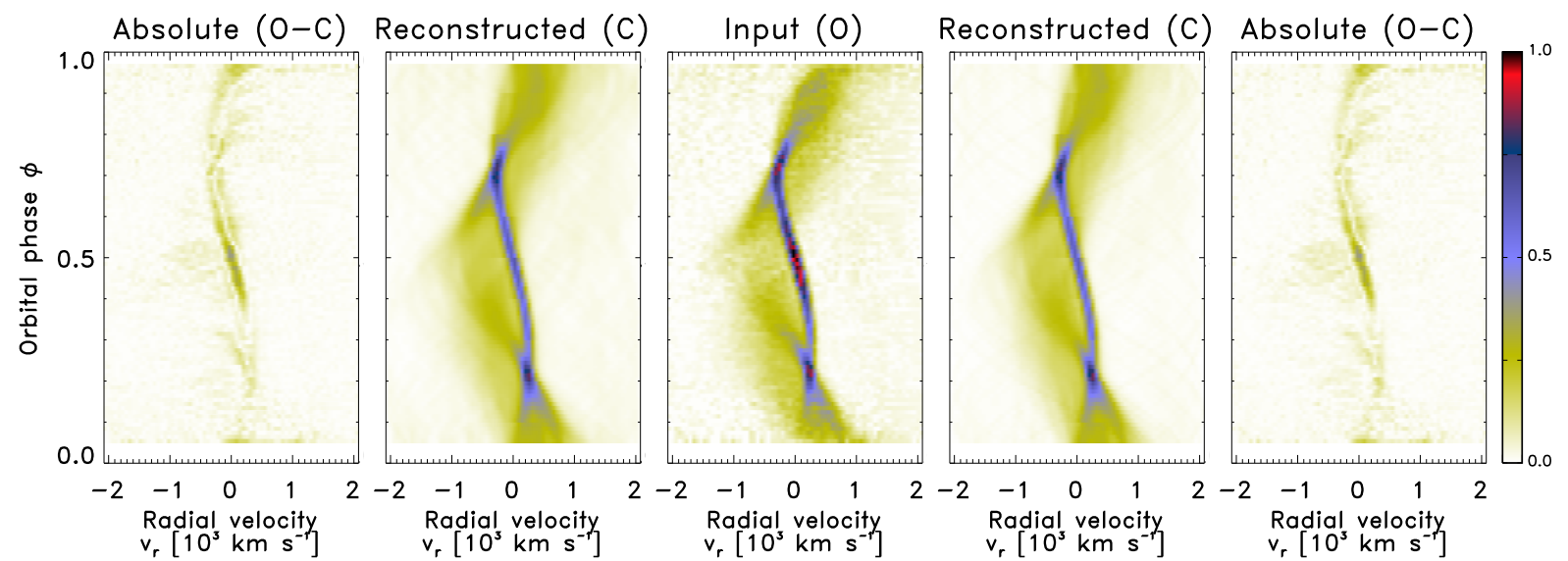

Fig. 5. Flux modulation mapping of HU Aqr. The standard and inside-out velocity maps are shown left and right, respectively. The top two panels show the modulation amplitude maps and the middle two panels show the phase of maximum flux maps. The phase of maximum flux map shows only pixels where the corresponding modulation amplitude is at least $7.5 \%$ of the maximum amplitude. This map is colour coded to represent phase: black (0.0), red (0.25), green (0.5) and blue (0.75). The bottom middle panel shows the trailed input spectra. The panels to the left and right of the middle panel show the summed trailed reconstructed and absolute residual spectra for the corresponding 10 consecutive half-phase tomogram. The model velocity profile overlay shown in all the tomograms is the same as the one in Fig. 2.

as high as required, we see that the flux distribution in the two distinct emission components is significantly improved. This is especially apparent in the split of the two components between phases $0.5-1.0$. The absolute $\mathrm{O}-\mathrm{C}$ spectra of both projections 


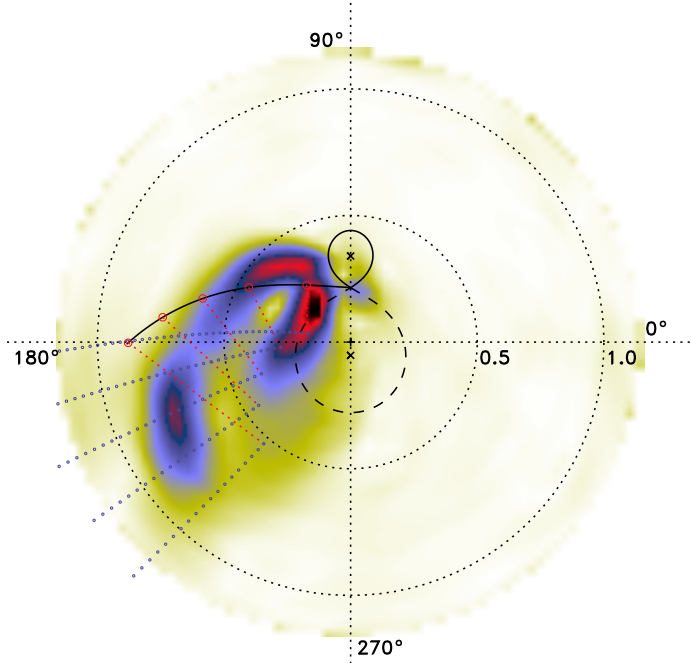

$(v, \theta)\left[10^{3} \mathrm{~km} \mathrm{~s}^{-1}\right.$, degrees $]$

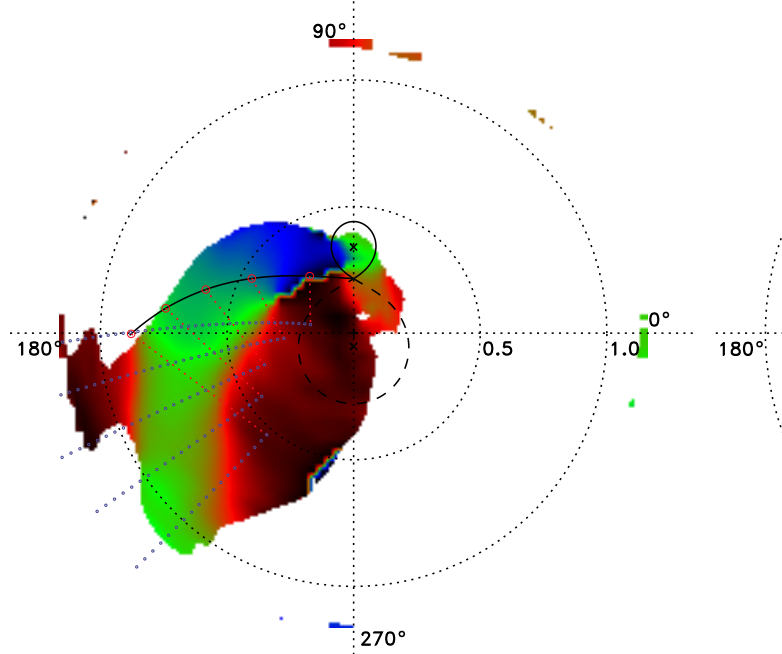

$(v, \theta)\left[10^{3} \mathrm{~km} \mathrm{~s}^{-1}\right.$, degrees $]$





$(\mathrm{v}, \theta)\left[10^{3} \mathrm{~km} \mathrm{~s}^{-1}\right.$, degrees $]$

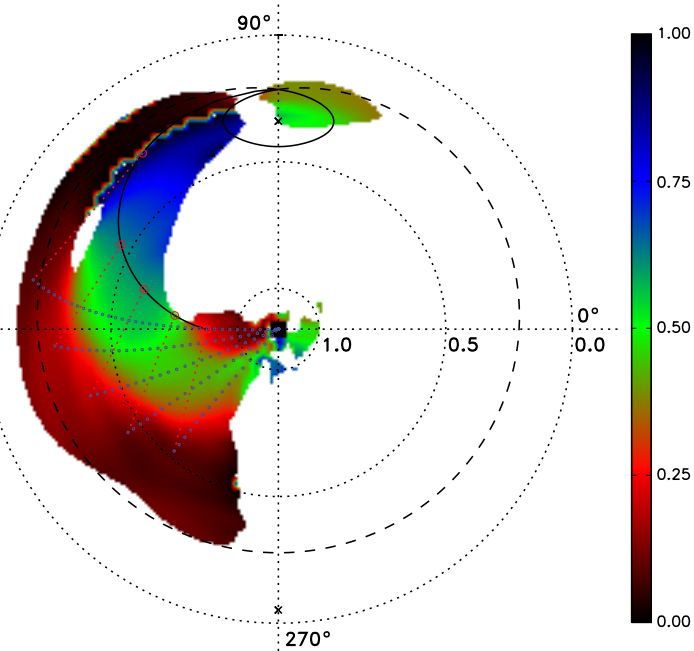

$(v, \theta)\left[10^{3} \mathrm{~km} \mathrm{~s}^{-1}\right.$, degrees $]$

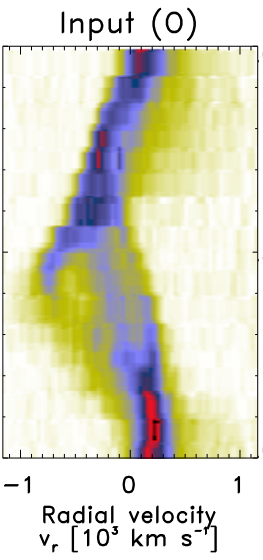

Reconstructed (C) Absolute (O-C)

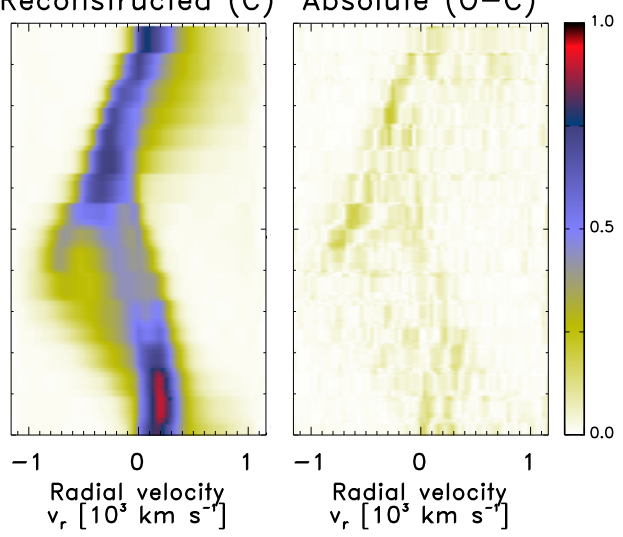

Fig. 6. Flux modulation mapping of V834 Cen. Same layout as Fig. 5. The model velocity profile overlay shown in all the tomograms is the same as the one in Fig. 3.

clearly show the improvement in the reproduction of the input spectra with rms values of 0.070 and 0.066 compared to the nonmodulated rms values of 0.094 and 0.092 .

We see in both the standard and inside-out amplitude maps that most of the emission components seen in the Doppler tomograms are heavily flux modulated. In the standard amplitude map the emission associated with the lower accretion curtain (represented by the spot in the upper right quadrant) appears to be the most flux modulated component. The emission associated with the upper accretion curtain (represented by the spot in the lower left quadrant) is also flux modulated, but it is split into two modulating components. In the inside-out amplitude map this emission is also split into two components, but now they appear to be the most modulated. 


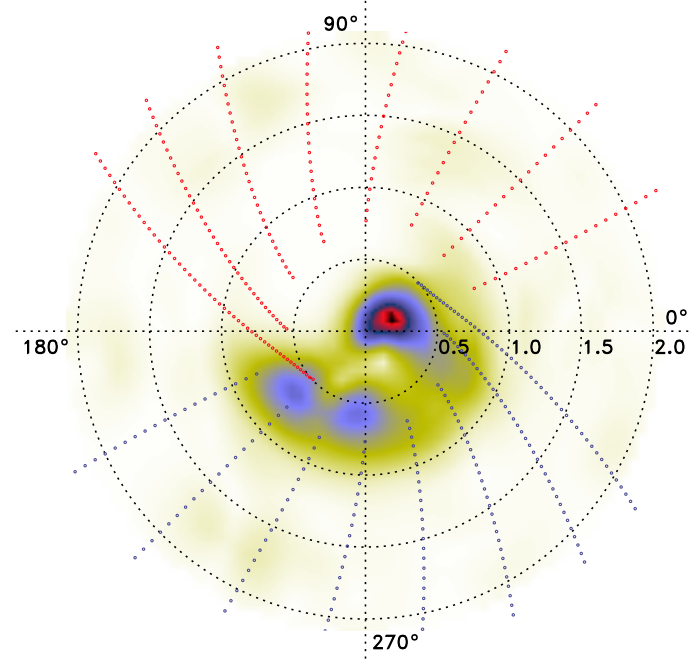

$(\mathrm{v}, \theta)\left[10^{3} \mathrm{~km} \mathrm{~s}^{-1}\right.$, degrees $]$

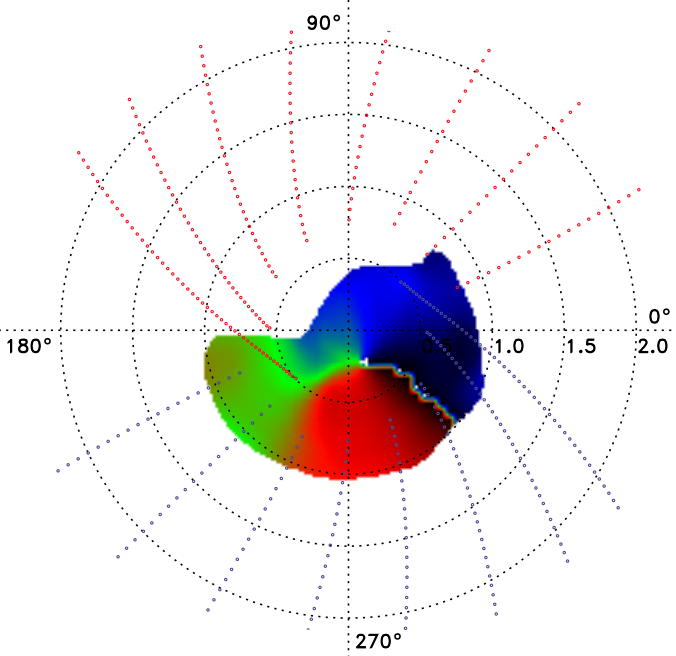

$(\mathrm{v}, \theta)\left[10^{3} \mathrm{~km} \mathrm{~s}^{-1}\right.$, degrees $]$


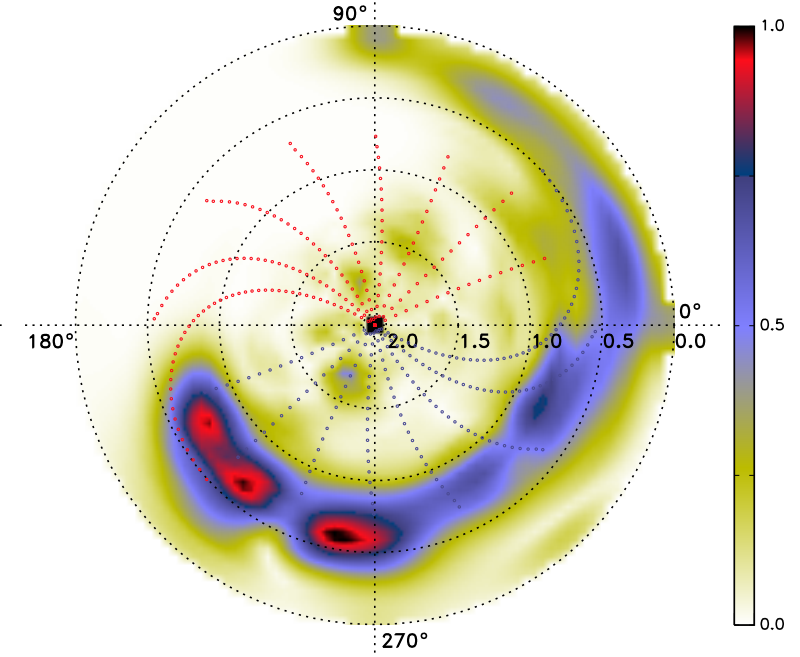

$(\mathrm{v}, \theta)\left[10^{3} \mathrm{~km} \mathrm{~s}^{-1}\right.$, degrees $]$

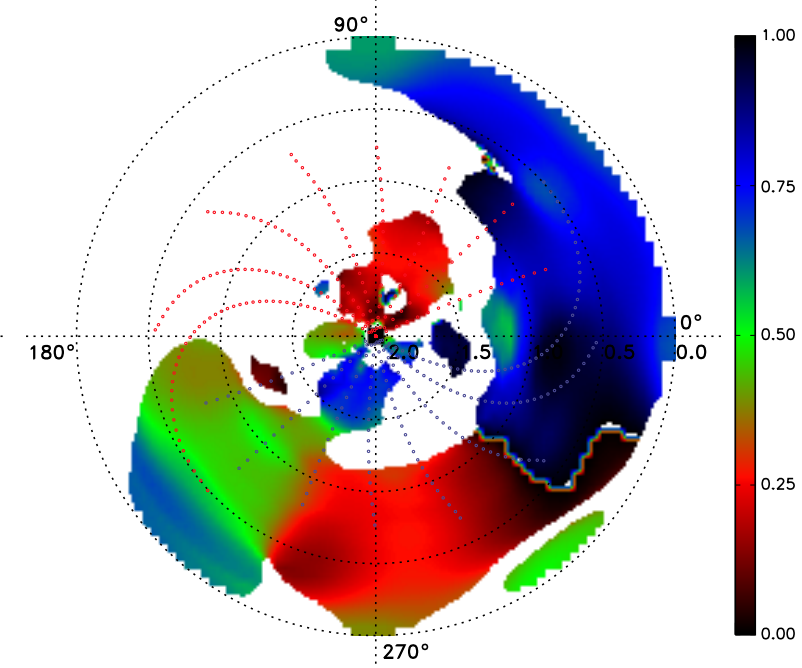

$(\mathrm{v}, \theta)\left[10^{3} \mathrm{~km} \mathrm{~s}^{-1}\right.$, degrees $]$
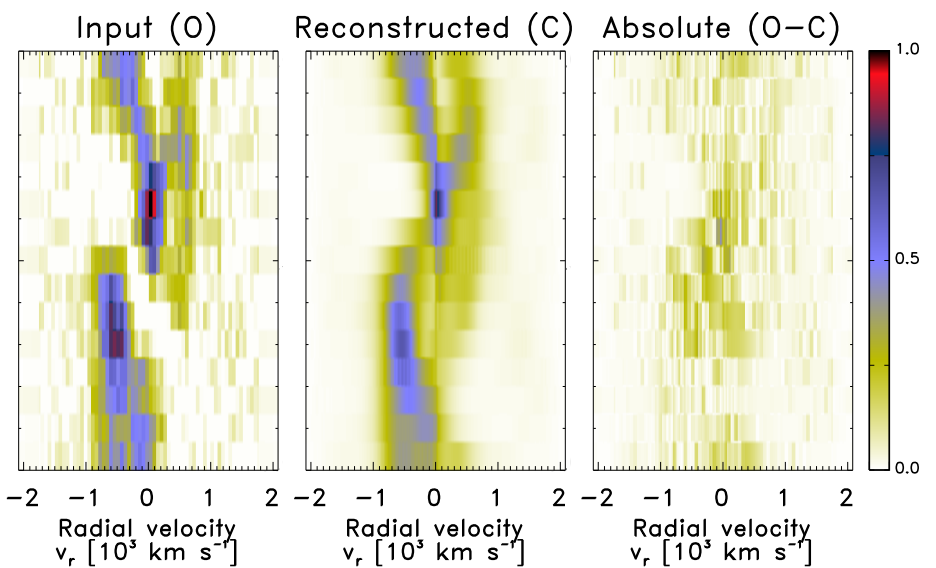

Fig. 7. Flux modulation mapping of PQ Gem. Same layout as Figs. 5 and 6 . The model velocity profile overlay shown in all the tomograms is the same as the one in Fig. 4.

In both the standard and inside-out phase of maximum flux maps we see that the proposed upper curtain is mostly green, which means that it appears brightest at spin phase 0.5 when we have a lateral view of the curtain. In the inside-out map the low $\left(<500 \mathrm{~km} \mathrm{~s}^{-1}\right)$ and high $\left(>1500 \mathrm{~km} \mathrm{~s}^{-1}\right)$ parts of the proposed upper curtain are blue. This implies that the "start" (as it leaves the orbital plane) and "end" (as it falls onto the primary) of the curtain appear brightest at spin phase 0.75 when these parts flow, respectively, towards and away from us. The proposed lower curtain is mostly blue in both phase of maximum flux maps, that is to say, it appears brightest at spin phase 0.75 when the curtain is predominantly flowing towards us. In the inside-out map the 
high velocity ( $>1200 \mathrm{~km} \mathrm{~s}^{-1}$ ) part of the proposed lower curtain (as it falls onto the primary) is red, which means that it appears brightest at spin phase 0.25 when this part is predominantly flowing away from us. The arc of emission running through the lower right quadrant that "connects" the proposed upper and lower curtains is mostly black and red, in other words, it appears brightest around spin phases $0.0-0.25$.

\section{Summary}

We applied our inside-out velocity projection to published data of the polars HU Aqr, V834 Cen and the intermediate polar PQ $\mathrm{Gem}$. We note that the inside-out projection leads to a redistribution in the relative contrast levels in and amongst emitting components such as the ballistic and magnetically confined accretion flows. The inside-out projection exposes low velocity emission details which are overly compacted in the standard projection. Similarly, it enhances high velocity emission details which are washed out in the standard projection.

In the inside-out tomogram of HU Aqr the ballistic accretion stream appears discernible to one and a quarter times the velocity extent observed for it in the standard tomogram. The blended emission from the secondary and ballistic stream seen in the standard tomogram of V834 Cen is more separated in the insideout tomogram. Also, the separation between the lower velocity emission from the ballistic accretion stream and that from the magnetically confined accretion is more pronounced compared to the standard tomogram. More striking in the inside-out tomogram, however, is the funnelling of the emission from the magnetically confined accretion flow as it falls towards the primary. As expected, the inside-out tomogram of PQ Gem shows an extended projection of the lower velocity $\left(<1000 \mathrm{~km} \mathrm{~s}^{-1}\right)$ emission associated with the accretion curtains. These results, especially for HU Aqr and V834 Cen, show that the inside-out projection has the ability to reveal emission not discernible in the standard projection. Any extra emission information obtained with the inside-out projection has the potential to help us form a more complete picture of the emission components in these types of systems.

We also applied our flux modulation mapping technique, in both the standard and inside-out projections, to the above mentioned test cases. We found that the information obtained through the amplitude of the phased modulation and the phase of maximum observed flux neatly confirms the current expectation of how the emission components in the test cases modulate over an observed orbital or spin phase.

We conclude that the inside-out projection complements the existing standard projection. Together with flux modulation mapping, it forms a useful addition for unravelling the different emission line components in the observed spectra of mCVs. In the spirit of open access, we are making our code publicly accessible. It can be downloaded from the CDS and http://www . saao.ac . za/ ejk/doptomog/main.html

Acknowledgements. We thank Axel Schwope and Coel Hellier for providing the HU Aqr and PQ Gem data, respectively. Also, we thank the referee for the constructive comments. This material is based upon work supported financially by the National Research Foundation. Any opinions, findings and conclusions or recommendations expressed in this material are those of the author(s) and therefore the NRF does not accept any liability in regard thereto.

\section{References}

Čechura, J., Vrtilek, S. D., \& Hadrava, P. 2015, MNRAS, 450, 2410 Ferrario, L., de Martino, D., \& Gänsicke, B. T. 2015, Space Sci. Rev., 191, 111 Heerlein, C., Horne, K., \& Schwope, A. D. 1999, MNRAS, 304, 145 Hellier, C. 1997, MNRAS, 288, 817

Hellier, C. 1999, ApJ, 519, 324

Hellier, C. 2001, Cataclysmic Variable Stars - How and Why They Vary (Springer-Praxis)

Kotze, E. J., Potter, S. B., \& McBride, V. A. 2015, A\&A, 579, A77

Marsh, T. R. 2001, in Astrotomography, Indirect Imaging Methods in Observational Astronomy, eds. H. M. J. Boffin, D. Steeghs, \& J. Cuypers (Berlin: Springer Verlag), Lect. Notes Phys. 573, 1

Marsh, T. R., \& Horne, K. 1988, MNRAS, 235, 269

Peris, C. S., Vrtilek, S. D., Steiner, J. F., et al. 2015, MNRAS, 449, 1584

Potter, S. B., Cropper, M., Mason, K. O., Hough, J. H., \& Bailey, J. A. 1997, MNRAS, 285, 82

Potter, S. B., Romero-Colmenero, E., Watson, C. A., Buckley, D. A. H., \& Phillips, A. 2004, MNRAS, 348, 316

Potter, S. B., Kotze, E. J., \& McBride, V. A. 2016, Proc. Sci., in press

Schwope, A. D., Thomas, H.-C., Beuermann, K., \& Reinsch, K. 1993, A\&A, 267, 103

Schwope, A. D., Mantel, K.-H., \& Horne, K. 1997, A\&A, 319, 894

Schwope, A. D., Catalán, M. S., Beuermann, K., et al. 2000, MNRAS, 313, 533

Šimić, D., Barwig, H., Bobinger, A., Mantel, K.-H., \& Wolf, S. 1998, A\&A, 329, 115

Spruit, H. C. 1998, ArXiv e-prints [arXiv: astro-ph/9806141]

Steeghs, D. 2003, MNRAS, 344, 448

Warner, B. 2003, Cataclysmic Variable Stars (Cambridge University Press) 